Vanessa Moraes Bezerra ${ }^{1}$ Danielle Souto de Medeiros ${ }^{1}$ Karine de Oliveira Gomes ${ }^{1}$ Raquel Souzas ${ }^{1}$

Luana Giatti ${ }^{2}$

Ana Paula Steffens ${ }^{1}$

Clavdia Nicolaevna Kochergin ${ }^{1}$

Cláudio Lima Souza ${ }^{1}$

\title{
Inquérito de Saúde em Comunidades Quilombolas de Vitória da Conquista, Bahia, Brasil (Projeto COMQUISTA): aspectos metodológicos e análise descritiva
}

Health survey in Quilombola communities (descendants of Afro-Brazilian slaves who escaped from slave plantations that existed in Brazil until abolition in 1888) in Vitória da Conquista in the state of Bahia (COMQUISTA Project), Brazil: methodological aspects and descriptive analysis

\author{
Cristiano Soares de Moura ${ }^{3}$ \\ Daniela Arruda Soares \\ Luis Rogério Cosme Silva Santos ${ }^{1}$ \\ Luiz Gustavo Vieira Cardoso ${ }^{1}$ \\ Márcio Vasconcelos de Oliveira ${ }^{1}$ \\ Poliana Cardoso Martins ${ }^{1}$ \\ Orlando Sílvio Caires Neves ${ }^{1}$ \\ Mark Drew Crosland Guimarães ${ }^{2}$
}

${ }^{1}$ Instituto Multidisciplinar em Saúde, Campus Anísio Teixeira, Universidade Federal da Bahia (IMS/CAT UFBA). R. Rio de Contas 58/Quadra 17/Lote 58 Candeias. 45.029-094 Vitória da Conquista BA Brasil.

vanessaenut@yahoo.com.b ${ }^{2}$ Programa de PósGraduação em Saúde Pública, Departamento de Medicina Preventiva Social, Faculdade de Medicina, Universidade Federal de Minas Gerais ${ }^{3}$ Division of Clinical Epidemiology, McGill University Health Center, McGill University.
Abstract The scope of this article was to present the methodology, preliminary descriptive results and the reliability of the instruments used in the COMQUISTA Project. It involved a cross-sectional study with adults ( $\geq 18$ years) and children (up to 5 years old) of Quilombola communities in Vitória da Conquista, Bahia. Data collection consisted of individual and household interviews, anthropometric and blood pressure measurements. A semi-structured questionnaire adapted from the Brazilian National Health Survey (PNS) was used and the interviews were conducted using handheld computers. 397 housing units were visited and 797 adults and 130 children were in terviewed. The demographic profile of the Quilombolas was similar to the Brazilian population with respect to sex and age, however, they had precarious access to basic sanitation and a low socioeconomic status. The analysis of reliability revealed the adequacy of strategies adopted for quality assurance and control in the study. The methodology used was considered adequate to achieve the objectives and can be used in other populations. The results indicate the need for implementing strategies to improve the quality of life and reduce the degree of vulnerability of the Quilombolas.

Key words Epidemiological surveys, Quilombola, Vulnerable communities, Health inequalities
Resumo O objetivo deste artigo foi apresentar a metodologia, resultados descritivos preliminares e a confiabilidade dos instrumentos utilizados no Projeto COMQUISTA. Estudo transversal com indivíduos adultos ( $\geq 18$ anos) e crianças (até cinco anos) de comunidades quilombolas de Vitória da Conquista, Bahia. A coleta de dados compreendeu a realização de entrevistas individuais e domiciliares, aferição de medidas antropométricas e pressão arterial. Utilizou-se o questionário semiestruturado da Pesquisa Nacional de Saúde (PNS), adaptado para a população quilombola, e as entrevistas foram realizadas em computadores portáteis. Foram visitados 397 domicílios e entrevistados 797 adultos e 130 crianças. O perfil demográfico dos quilombolas foi semelhante ao dos brasileiros quanto ao sexo e idade, porém, eles apresentaram precário acesso ao saneamento básico e baixo nível socioeconômico. A análise de confiabilidade demonstrou a adequação das estratégias adotadas para garantia e controle de qualidade no estudo. A metodologia empregada foi considerada adequada para o alcance dos objetivos e pode ser utilizada em outras populações. Os resultados sugerem a necessidade da implantação de estratégias para melhorar a qualidade de vida e reduzir o grau de vulnerabilidade dos quilombolas.

Palavras-chave Inquéritos epidemiológicos, Quilombola, Comunidades vulneráveis, Desigualdades em saúde 


\section{Introdução}

Inquéritos populacionais apresentam potencialidades para o estudo e o monitoramento das desigualdades sociais, bem como para o conhecimento do panorama nacional em saúde, seja em relação à avaliação dos seus determinantes ou para o estudo do acesso e utilização dos serviços de saúde. Além disso, as informações produzidas por esse tipo de estudo permitem correlacionar os resultados com as condições de vida da população, conhecer a distribuição dos fatores de risco e, ainda, verificar a percepção das pessoas a respeito do seu estado de saúde e o grau de satisfação com os serviços ${ }^{1}$.

De modo substancial, a dimensão étnico-racial vem adquirindo cada vez mais importância na área da saúde, tanto no âmbito da produção nacional como internacional ${ }^{2-5}$. Estudos demonstram que as iniquidades em saúde estão intimamente relacionadas à questão étnico-racial, evidenciando desigualdades nos perfis de adoecimento e morte de indivíduos que identificam sua cor da pele como pretos, pardos e indígenas ${ }^{6-11}$. Em algumas pesquisas o controle das variáveis socioeconômicas confirma o efeito independente da raça/cor e etnia sobre a saúde e demonstra a influência que o pertencimento a determinado grupo étnico-racial pode ter sobre o estado de saúde ${ }^{12,13}$.

É útil esclarecer que a variável raça/cor e etnia compõe um dos elementos junto a outros fatores do contexto político, socioeconômico, cultural e ambiental, expressando um conjunto complexo de determinações que nem sempre é captado por adequado tratamento estatístico nas pesquisas populacionais ${ }^{7,9,13}$. Deste modo, a noção sobre raça/cor e etnia deve ser compreendida como construção local, histórica e culturalmente determinada ${ }^{2,5}$.

No Brasil, a classificação étnico-racial oficialmente adotada corresponde ao quesito "raça/ cor" do censo demográfico do Instituto Brasileiro de Geografia e Estatística (IBGE), no qual os indivíduos se classificam em cinco categorias: branca, preta, parda, amarela e indígena ${ }^{5}$, critério que tem sido amplamente empregado ${ }^{7,14,15}$.

Embora seja imprescindível o aprofundamento da questão da diversidade étnico-racial da população brasileira nas pesquisas epidemiológicas ${ }^{5}$, igualmente, faz-se necessário extrapolar o diagnóstico de saúde do ponto de vista étnicoracial para além da análise de dados secundários, e buscar conhecer a realidade de grupos minoritários ainda não cobertos pelos sistemas de informações de saúde, tais como a população quilombola.

As comunidades remanescentes de quilombos são "grupos étnico-raciais, segundo critérios de autoatribuição, com trajetória histórica própria, dotados de relações territoriais específicas, com presunção de ancestralidade negra"16. Os quilombolas se distinguem por sua identidade étnica, pela forma particular de sua organização social, e pela predominante localização rural ${ }^{17}$. $\mathrm{O}$ processo histórico de escravização vivenciado por esta população trouxe como consequências desigualdades sociais e de saúde e, ainda, o acesso dificultado a bens e serviços ${ }^{18}$. Não obstante, estudos sobre a análise da situação de saúde das comunidades quilombolas ainda são escassos no Brasil, sendo identificadas apenas duas publicações de resultados de inquéritos de base populacional $^{19,20}$.

De forma geral, as pesquisas realizadas em comunidades quilombolas demonstram alta prevalência de problemas básicos de saúde ligados às precárias condições de vida e moradia, à ausência de saneamento básico e ao acesso restrito à educação e serviços de saúde ${ }^{17-21}$. Tal realidade expressa a vulnerabilidade da população quilombola no Brasil e evidencia a necessidade da realização de estudos epidemiológicos representativos que melhor caracterizem a situação de saúde, particularmente no Estado da Bahia, que possui um grande número de comunidades reconhecidas.

Encontra-se em fase de planejamento a Pesquisa Nacional de Saúde (PNS) ${ }^{22}$, de base domiciliar e em nível nacional, prevista para ser realizada em futuro próximo. A PNS foca principalmente em morbidade, estilos de vida e uso de serviços de saúde, primordialmente em âmbito urbano, tendo sido os instrumentos testados e disponibilizados ${ }^{22}$. Embora as informações referentes ao nível nacional não tenham poder para realizar inferências locais, a elaboração de instrumentos padronizados favorece o seu uso em distintos níveis e a realização de inquéritos em amostras populacionais específicas, permitindo assim uma melhor comparabilidade dos resultados $^{1}$. Diante do exposto, foi realizado um inquérito de saúde em comunidades quilombolas do município de Vitória da Conquista, Bahia (Projeto COMQUISTA) ${ }^{23}$, para avaliar as condições de vida, a situação de saúde, o acesso e o uso de serviços de saúde pela população residente. Para este inquérito optou-se por utilizar os instrumentos disponibilizados pela PNS, adaptando-os para a realidade predominante rural dessas comunidades. O objetivo deste artigo é apre- 
sentar a metodologia geral e os resultados descritivos preliminares do Projeto COMQUISTA.

\section{Métodos}

\section{População de estudo}

Trata-se de um estudo de delineamento transversal realizado em 2011. A população foi constituída por indivíduos com 18 anos ou mais e crianças com até cinco anos de idade residentes em comunidades quilombolas certificadas pela Fundação Palmares ${ }^{24}$ do município de Vitória da Conquista (BA). O projeto foi aprovado pelos Comitês de Ética e Pesquisa da Faculdade São Francisco de Barreiras e da Universidade Federal de Minas Gerais, todos os participantes adultos e os responsáveis pelas crianças menores de cinco anos assinaram os Termos de Consentimento Livre e Esclarecido (TCLE).

Vitória da Conquista registra 25 comunidades quilombolas certificadas pela Fundação Palmares, sediadas em cinco distritos da região, segundo informações coletadas por uma lista cedida pela Secretaria Municipal da Saúde ${ }^{25}$. Para se garantir representatividade e viabilidade da pesquisa, optou-se por uma estratégia amostral considerando a extensão territorial e o tamanho das comunidades, uma vez que algumas destas tinham poucos domicílios e, por questões logísticas, foram excluídas do estudo. Sendo assim, os princípios amostrais utilizados foram: a) selecionar uma comunidade por distrito; b) incluir somente comunidades com pelo menos 50 famílias cadastradas; c) selecionar domicílios; d) convidar todos os adultos (18 ou mais anos) e crianças $<5$ anos residentes no domicílio sorteado.

Para efeito de cálculo amostral, considerouse somente a população elegível de adultos. Como não havia informações em relação ao número de crianças nas comunidades, optou-se por entrevistar todas as crianças dos domicílios sorteados a participar da pesquisa. A população de adultos foi estimada em 2.935 indivíduos com 18 ou mais anos, oriundos de 10 comunidades que apresentaram pelo menos 50 famílias cadastradas. Foram considerados os seguintes parâmetros: a) prevalência, a priori, de $50 \%$, dado a heterogeneidade dos eventos a serem mensurados; b) precisão de 5\%; c) nível confiança de 95\%; d) efeito de desenho igual a 2 ; em função da média de dois adultos por domicílios na zonal rural do Brasil estimada pelo IBGE; e, e) $30 \%$ de perdas, definida após realização do piloto, totalizando 884 indivíduos adultos a serem entrevistados.
O plano amostral foi realizado em dois estágios. Estágio 1: seleção aleatória de uma comunidade quilombola em cada distrito, considerando-se o tamanho populacional de cada uma, totalizando cinco comunidades (Corta-Lote, $\mathrm{Ma}-$ ria de Clemência, Furadinho, Lagoa de Melquíades e Boqueirão). Após enumerar a população total de cada distrito, ordenada pelas comunidades distritos, foi sorteado um número aleatório entre um e a população total de cada distrito, e a comunidade cujo intervalo continha este número foi selecionada. Em dois distritos que tinham somente uma comunidade, esta foi selecionada. Estágio 2: Seleção aleatória dos domicílios de acordo com a distribuição proporcional de domicílios por distrito. Todos os adultos e crianças $<5$ anos de cada domicílio foram convidados a participar.

\section{Instrumentos}

O instrumento utilizado para a realização das entrevistas foi o questionário semiestruturado Inquérito Região Integrada do Distrito Federal (RIDE) da $\mathrm{PNS}^{22}$, composto por um bloco de informações sobre o domicílio e outro sobre o indivíduo. No questionário domiciliar, as questões estão divididas em sessões que investigam os seguintes pontos: 1) contatos com o domicílio; 2) lista de moradores do domicílio; 3) relação de parentesco entre os moradores; 4) situação socioeconômica; 5) rendimentos (homens e mulheres); 6) informações do domicílio; 7) características contextuais (vizinhança); 8) características de saúde dos moradores do domicílio; 9) cobertura de plano de saúde; 10) gastos com saúde; e, 11) utilização de serviços de saúde.

No questionário individual as questões estão distribuídas em 10 blocos de informações: A) características sociodemográficas e apoio social (a variável "cor/raça" é autorreferida e categorizada em: branca, preta, parda, amarela e indígena, de acordo com o proposto pelo IBGE); B) autoavaliação do estado de saúde; C) estilo de vida; D) morbidade referida; E) acidentes e violência; F) saúde da mulher; G) crianças menores de dois anos; H) saúde bucal; I) saúde dos idosos; e, J) desempenho do sistema de saúde.

O questionário sofreu adaptações com o objetivo de retratar a realidade de uma comunidade quilombola da zona rural e facilitar a compreensão por parte desta população, porém, houve a preocupação em manter ao máximo a estrutura original da PNS para garantir a comparabilidade das informações. No primeiro momento, foi realizada uma ampla discussão sobre o 
instrumento com um grupo de especialistas para avaliar cada questão e decidir sobre sua permanência ou não. Também foram feitas as adaptações em termos de vocabulário e/ou opções de respostas, considerando o contexto em que o questionário seria aplicado. Desse modo, foram introduzidos alguns termos como "baba", expressão que caracteriza o futebol na região, e "açúcar no sangue" para esclarecer sobre a glicemia.

Em seguida, procedeu-se à realização dos prétestes e o instrumento foi aplicado pela equipe de pesquisadores para verificar sua adequação quanto a: 1) linguagem; 2) sequência e coerência entre as questões; 3 ) instruções sobre as questões a serem saltadas; e, 4) o tempo necessário para a aplicação da entrevista. Após esta etapa, foi realizada outra discussão com o grupo de especialistas para obtenção da versão a ser utilizada no estudo piloto. Após a realização do piloto, foram feitas novas modificações quanto à linguagem e incluídos termos como "aguada", "adobe", "pressão alta" e "derrame" para especificar "tanque/açude", "barro", "hipertensão" e "acidente vascular cerebral", respectivamente, e obteve-se a versão final do instrumento.

A maior alteração efetuada foi o resumo do questionário domiciliar, já que a proposta metodológica do Projeto COMQUISTA, diferente da PNS, considerava elegíveis a participarem do estudo todos os indivíduos residentes no domicílio com 18 ou mais anos de idade. Assim, algumas informações que seriam investigadas pelo módulo individual foram excluídas do módulo domiciliar: 1) relação de parentesco entre os moradores; 2) situação socioeconômica; 3 ) rendimentos; 4) cobertura de plano de saúde; e, 5) utilização de serviços de saúde. Em relação ao nível individual, a anemia foi incluída na lista das doenças investigadas no bloco sobre morbidades; a investigação referente às crianças foi estendida para a idade de até cinco anos e o módulo de saúde bucal foi excluído. O questionário final está disponível em http://projetocomquista.wordpress.com ${ }^{23}$.

Os questionários foram aplicados com o auxílio de computadores portáteis (HP Pocket Rx5710) e o software usado para a programação e armazenamento dos dados foi o Questionnaire Development System (QDS ${ }^{\mathrm{TM}}$; NOVA Research Company), versão 2.6.1. Este programa é composto por quatro aplicativos: 1) Questionnaire Design Studio para a construção do questionário; 2) CAPI; 3) HAPI para a visualização dos questionários; e, 4) Warehouse Manager para a organização, o armazenamento e o gerenciamento do banco de dados.
Também foram utilizados equipamentos de medidas antropométricas para adultos e crianças e de aferição da pressão arterial (PA) somente para adultos. O peso foi verificado em balança eletrônica portátil Marca Marte ${ }^{\circledR}$, Modelo LC200PP, com capacidade até $200 \mathrm{Kg}$ e sensibilidade de $50 \mathrm{~g}$, utilizando uma bandeja de pesagem pediátrica Marca Seca ${ }^{\circledR}$, Modelo 522-501100090, para pesar crianças menores de dois anos.

Em adultos e crianças com dois anos ou mais, a altura foi obtida por meio de um estadiômetro portátil e transportável da marca CauMaq ${ }^{\circledR}$, modelo EST-22, com capacidade de 300 a 2.000 $\mathrm{mm}$. Em crianças menores de dois anos, a altura foi medida utilizando um estadiômetro pediátrico, modelo esteira, portátil, marca Sanny ${ }^{\circledR}$, com escala de 20 a $105 \mathrm{~cm}$ e resolução de $0,5 \mathrm{~cm}$. A circunferência da cintura foi coletada por meio de uma fita de medida antropométrica da marca Cardiomed ${ }^{\circledR}$, modelo T-87-Wiso, com retração automática, trava na extremidade da fita e capacidade de $2 \mathrm{~m}$ de comprimento. Por fim, a PA foi aferida por esfigmomanômetro eletrônico digital, marca Omron ${ }^{\circledR}$, modelo HEM-742.

\section{Piloto}

O estudo piloto foi realizado em uma comunidade quilombola não selecionada para a pesquisa e decidiu-se pela aplicação do instrumento em um número equivalente a $8 \%$ da amostra do estudo principal. Os principais objetivos do piloto foram avaliar: 1) instrumentos de coleta de dados; 2) procedimentos para obtenção do TCLE; 3) grau de participação; 4) protocolos operacionais; 5) plano amostral; 6) dimensionamento do tempo despendido com transporte; 7) abordagem e aplicação das entrevistas; 8) composição das equipes para execução da coleta de dados; e, 9) identificar potenciais situações diferentes daquelas previstas inicialmente.

\section{Sensibilização}

O projeto foi apresentado na reunião mensal do Conselho Regional Quilombola, oportunidade em que estavam presentes as lideranças das comunidades, visando obter o apoio dos mesmos para divulgação e realização da pesquisa. Além disso, foi estabelecido contato com representantes das Equipes de Saúde da Família e Agentes Comunitários de Saúde (ACS) dos distritos sorteados. A sensibilização nos domicílios ocorreu durante o mapeamento das comunidades, quando a equipe e os ACS informavam direta- 
mente aos moradores que estavam presentes nos domicílios sobre a pesquisa a ser realizada. Foi entregue material impresso de divulgação e ainda coletadas informações como número e idade dos moradores do domicílio para identificação do número de elegíveis.

\section{Mapeamento}

O mapeamento foi realizado pela delimitação territorial a partir de estradas vicinais e marcação de pontos relativos a domicílios e equipamentos da comunidade (estabelecimentos comerciais e de saúde, igreja, campo de futebol etc.), obtidos em frente à porta principal dos locais. Foram utilizados localizador GPS (Global Positioning System), com precisão de $10 \mathrm{~m}$, e máquina fotográfica digital.

Simultaneamente à marcação por GPS, realizou-se a identificação através de anotações de cada ponto (número e coordenadas geográficas) gerado pelo GPS, em ordem numérica, a partir de um marcador de início do limite da comunidade (ponto um). Ao mesmo tempo era realizada a documentação fotográfica. Os dados foram transferidos para computador e organizados no software Microsoft Excel 2010.

Para confecção dos mapas, os dados brutos do GPS foram transferidos para o programa GPS TrackMaker, versão 13.8, em seguida exportados para o Quantum GIS, versão 1.7.026. Os pontos localizados após os domicílios da extremidade foram considerados como vértices/limites.

\section{Treinamento}

Para realização das entrevistas foram selecionados graduandos da Universidade Federal da Bahia. As seguintes estratégias foram adotadas para o treinamento dos entrevistadores: dramatização de entrevistas; manuseio dos pockets; aferição de medidas antropométricas e PA, e aplicação das entrevistas em indivíduos internos e externos à comunidade universitária. A padronização dos procedimentos de entrevistas e realização das medidas foi garantida pelo uso de manuais elaborados pelos pesquisadores. Após avaliação de desempenho, foram selecionados 24 entrevistadores.

\section{Coleta de dados}

Foram organizadas cinco equipes para a coleta dos dados, compostas por dois entrevistadores e estabelecida uma estrutura de escala diá- ria, com carga horária de $20 \mathrm{~h}$ semanais. Para cada comunidade havia uma equipe de três supervisores, constituída pelos pesquisadores do projeto e um coordenador de campo. Durante a abordagem dos domicílios foi utilizado um script visando padronizar e orientar os entrevistadores para o convite aos residentes. Após abordagem e convite foram aplicados os TCLE, seguidos das entrevistas com o uso dos pockets e finalização com as tomadas de medidas.

A coleta das medidas antropométricas foi baseada nas técnicas preconizadas por Jelliffe ${ }^{27} \mathrm{e}$ a aferição da PA foi realizada de acordo com a VI Diretrizes de Hipertensão Arterial da Sociedade Brasileira de Cardiologia ${ }^{28}$. Foram utilizados manuais específicos com instruções detalhadas sobre os procedimentos adequados para a coleta de cada medida. Os valores das medidas foram anotados em um cartão e entregues aos participantes. A realização das medidas após a aplicação das entrevistas foi adotada como procedimento padrão para garantir que o participante estivesse em repouso, critério para aferição da $\mathrm{PA}$, e otimizar o tempo necessário para montagem dos equipamentos.

Foi realizada supervisão direta da coleta dos dados e os entrevistadores tiveram sua produção controlada diariamente por meio de fichas de controles de visitas ao domicílio, controle de entrevistas e reentrevistas e controle de utilização dos pockets. Os supervisores de campo foram responsáveis pela contínua assistência aos entrevistadores na localização e abordagem prévia dos entrevistados e pela revisão permanente dos controles de campo. O controle das entrevistas era repassado à coordenação do campo e o de utilização dos pockets à central de banco de dados, sendo realizada diariamente a conferência dos dados coletados.

Por fim, para a avaliação da confiabilidade das respostas obtidas em entrevistas, foi prevista a realização de reentrevistas em $5 \%$ da amostra, que ocorreram no prazo máximo de uma semana após a realização da entrevista inicial.

\section{Análise estatística}

Foi feita análise descritiva de dados sociodemográficos da população e de algumas características dos domicílios. A comparação entre participantes e não participantes foi realizada pelo teste qui-quadrado, com nível de significância de $5 \%$. Para a análise de confiabilidade foram utilizadas a concordância observada e a estatística Kappa para variáveis categóricas, e o coeficiente 
de correlação de Pearson para variáveis contínuas $^{29}$. As estimativas foram obtidas com os programas Epi Info, versão 3.5.3, e R, versão 2.11.1.

\section{Resultados}

\section{Piloto}

O estudo piloto foi realizado na comunidade quilombola Sinzoca. Foram visitados 55 domicílios, dos quais 48 (87,3\%) participaram do estudo, as perdas ocorreram principalmente por não encontrar moradores após três visitas. Foram realizadas 97 entrevistas ( 91 de adultos e seis de crianças) e obteve-se $24,4 \%$ de perdas, valor superior ao inicialmente previsto de $20,0 \%$.

Foram realizadas reentrevistas em $37,1 \%$ dos indivíduos e a confiabilidade de algumas variáveis selecionadas como "sexo" e "cor/raça" apresentaram índice Kappa de 1,0 e 0,84, respectivamente, interpretadas como excelentes. As variáveis "diagnóstico de hipertensão arterial", "autoavaliação de saúde" e "frequência de consumo de bebidas alcoólicas" tiveram sua confiabilidade classificadas como substancial, moderada e razoável, respectivamente.

\section{Estudo Principal}

\section{Mapeamento}

Para cada comunidade foi desenvolvido um mapa, listas de domicílios com identificação visual e a respectiva correspondência geográfica, além da referência de um dos moradores e o número de elegíveis em cada domicílio. A Figura 1 apresenta a situação geográfica do município em relação ao Estado, das comunidades em relação à sede do município, e o exemplo de uma das comunidades (Corta-Lote), indicando a distribuição dos domicílios e equipamentos.

\section{Coleta de dados}

A coleta de dados ocorreu entre os meses de setembro e outubro de 2011, totalizando 36 dias de campo (média de 23 entrevistas/dia). Foram identificados 1082 indivíduos elegíveis para o estudo nos 422 domicílios sorteados, sendo 943
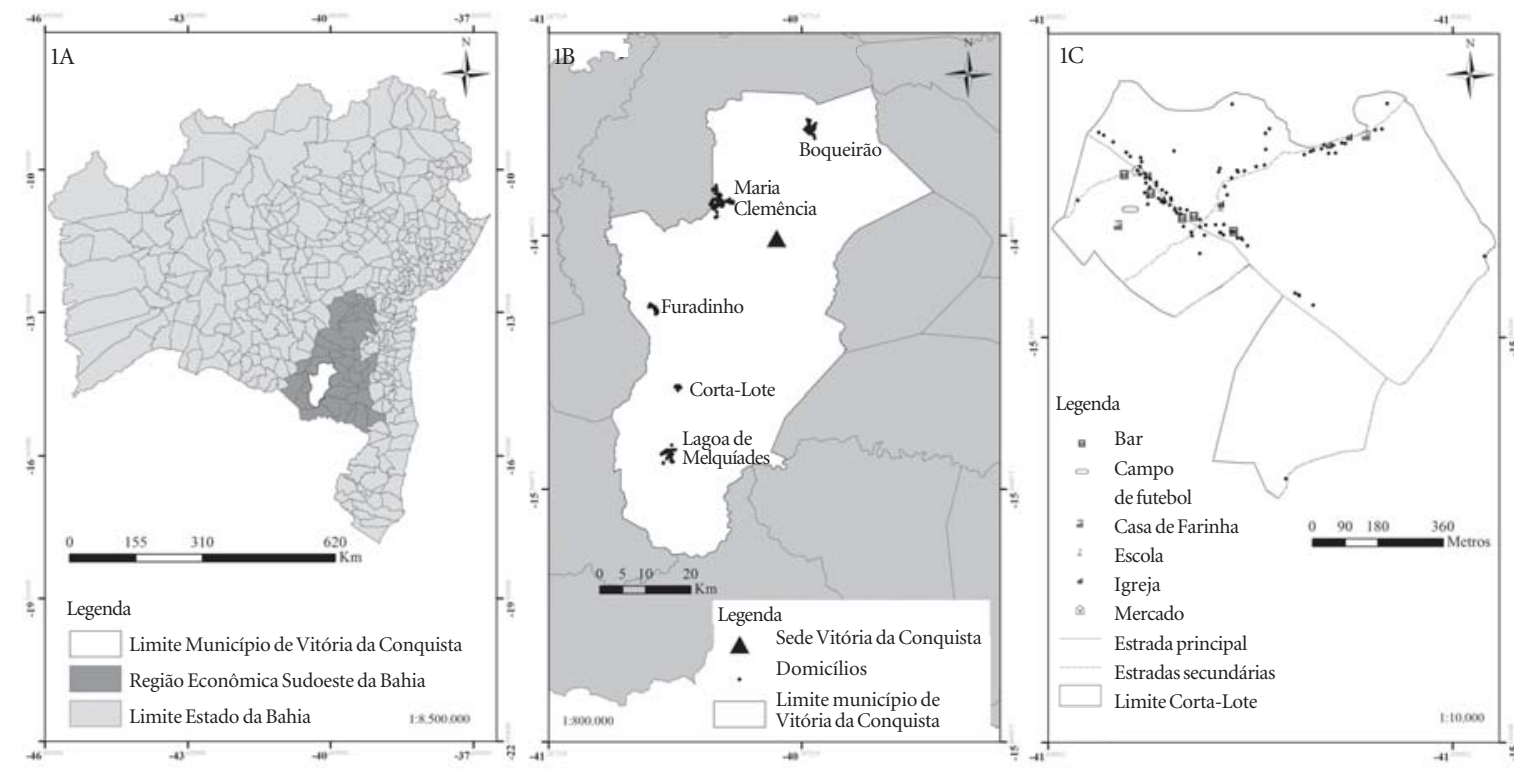

Projeção Transverse Mercator Datum WGS 1984

Edição: 09/10/2012

Figura 1. 1A. Divisão político-administrativa da Bahia, 2012; 1B. Município de Vitória da Conquista e Comunidades Quilombolas; 1C. Comunidade Quilombola Corta-Lote

Fontes: Comunidades Quilombolas: mapeamento Projeto COMQUISTA; Divisão Político-Administrativa da Bahia 2012: DIGEO/SEI Superintendência de Estudos Econômicos e Sociais da Bahia - Coordenação de Cartografia e Geoprocessamento - CARTGEO, Coordenação de Recursos Naturais - CRNA, Divisão de Informação Geoambientais. 
adultos e 139 crianças menores de cinco anos. A comunidade com maior proporção de elegíveis foi Maria de Clemência (34,7\% adultos; 38,8\% crianças), e aquelas com menor proporção foram Corta-lote para adultos (9,9\%) e Furadinho para crianças $(5,0 \%)$ (Tabela 1$)$.

Do total de domicílios visitados, 393 (93,1\%) participaram do estudo e a maioria das perdas $(96,5 \%)$ ocorreu por não se ter encontrado moradores após três visitas. Foram entrevistados 797 adultos e 130 crianças, com distribuição proporcional à observada na população de elegíveis das comunidades pesquisadas. $\mathrm{O}$ percentual de perdas de adultos foi de $15,5 \%$ e entre as crianças de 6,5\%, em ambos os casos, as maiores proporções de perdas foram observadas na comunidade Corta-lote (18,3\% e 19,0\% para adultos e crianças, respectivamente) (Tabela 1).

O principal motivo das perdas de entrevistas com adultos foi não ter encontrado os indivíduos após três visitas $(82,2 \%)$, enquanto que as recusas representaram 17,8\%. Do total de 146 adultos não entrevistados, foram obtidas informações adicionais de 115 indivíduos, que possibilitaram estabelecer comparações entre os grupos de participantes e não participantes. A perda foi significativamente maior em indivíduos do sexo masculino e com idade entre 18 e 34 anos (Tabela 2).

Reentrevistas e análise de confiabilidade

Foram realizadas 42 reentrevistas $(4,5 \%$ da amostra), as variáveis cuja confiabilidade foi analisada mostraram percentuais de concordância observada variando de 78,0 a 100,0\%, com índices Kappa de 0,60 a 1,00 (Tabela 3). Das 25 variáveis, $60,0 \%$ e $8,0 \%$ apresentaram excelente ou moderada concordância Kappa, respectivamente. As variáveis "idade" e "escolaridade" (anos completos de estudo) apresentaram alta consis- tência ao obterem coeficiente de correlação de Pearson igual a 0,97 e 0,96 , respectivamente. Por outro lado, a variável "número de dias sem trabalhar por motivo de saúde" apresentou valor igual a 0,53 .

\section{Características gerais da amostra}

Do total de domicílios investigados, $2,3 \%$ recebiam água da rede geral de abastecimento, $9,7 \%$ tinham esgotamento sanitário pela rede geral de esgoto ou fossa séptica, nenhum apresentou coleta regular como destino do lixo e $94,6 \%$ tinham energia elétrica. A maioria dos domicílios era visitada mensalmente pelos ACS, contudo, 25,8\% relataram nunca ter recebido visitas. Quanto à

Tabela 2. Comparação entre participantes e não-participantes segundo a comunidade, sexo e idade. Projeto COMQUISTA, Brasil, 2011.

\begin{tabular}{|c|c|c|c|c|}
\hline \multirow{2}{*}{ Variáveis } & \multicolumn{2}{|c|}{ Participantes } & \multicolumn{2}{|c|}{$\begin{array}{c}\text { Não } \\
\text { participantes }\end{array}$} \\
\hline & $\mathbf{n}$ & $\%$ & $\mathbf{n}$ & $\%$ \\
\hline \multicolumn{5}{|l|}{ Comunidade } \\
\hline Corta-lote & 76 & 9,5 & 17 & 11,6 \\
\hline Clemência & 270 & 33,9 & 57 & 39,0 \\
\hline Furadinho & 158 & 19,8 & 16 & 11,0 \\
\hline L. Melquíades & 136 & 17,1 & 24 & 16,4 \\
\hline Boqueirão & 157 & 19,7 & 32 & 22,0 \\
\hline \multicolumn{5}{|l|}{ Sexo } \\
\hline Masculino & 364 & 45,7 & 89 & 77,4 \\
\hline Feminino & 433 & 54,3 & 26 & $22,6^{*}$ \\
\hline \multicolumn{5}{|l|}{ Idade } \\
\hline 18 a 34 anos & 289 & 36,3 & 75 & 65,2 \\
\hline 35 a 59 anos & 331 & 41,5 & 33 & 28,7 \\
\hline 60 anos ou mais & 177 & 22,2 & 7 & $6,1^{*}$ \\
\hline
\end{tabular}

* $<0,05$ - qui-quadrado de tendência linear.

Tabela 1. Síntese geral de recrutamento do estudo. Projeto COMQUISTA, Brasil, 2011.

\begin{tabular}{|c|c|c|c|c|c|c|c|c|c|c|c|c|}
\hline \multirow{3}{*}{ Comunidade } & \multicolumn{4}{|c|}{ Indivíduos elegíveis } & \multicolumn{4}{|c|}{ Entrevistas realizadas } & \multicolumn{4}{|c|}{ Perdas $^{*}$} \\
\hline & \multicolumn{2}{|c|}{ Adultos } & \multicolumn{2}{|c|}{ Crianças } & \multicolumn{2}{|c|}{ Adultos } & \multicolumn{2}{|c|}{ Crianças } & \multicolumn{2}{|c|}{ Adultos } & \multicolumn{2}{|c|}{ Crianças } \\
\hline & $\mathbf{n}$ & $\%$ & $\mathbf{n}$ & $\%$ & $\mathbf{n}$ & $\%$ & $\mathbf{n}$ & $\%$ & $\mathbf{n}$ & $\%$ & $\mathbf{n}$ & $\%$ \\
\hline Corta-lote & 93 & 9,9 & 21 & 15,1 & 76 & 9,5 & 17 & 13,1 & 17 & 18,3 & 4 & 19,0 \\
\hline Maria de Clemência & 327 & 34,7 & 54 & 38,8 & 270 & 33,9 & 51 & 39,2 & 57 & 17,4 & 3 & 5,6 \\
\hline Furadinho & 174 & 18,5 & 7 & 5,0 & 158 & 19,8 & 6 & 4,6 & 16 & 9,2 & 1 & 14,3 \\
\hline Lagoa de Melquíades & 160 & 17,0 & 30 & 21,6 & 136 & 17,1 & 30 & 23,1 & 24 & 15,0 & 0 & 0,0 \\
\hline Boqueirão & 189 & 20,0 & 27 & 19,4 & 157 & 19,7 & 26 & 20,0 & 32 & 16,9 & 1 & 3,7 \\
\hline Total & 943 & 100,0 & 139 & 100,0 & 797 & 100,0 & 130 & 100,0 & 146 & 15,5 & 9 & 6,5 \\
\hline
\end{tabular}

* Calculadas em relação ao total de indivíduos elegíveis. 


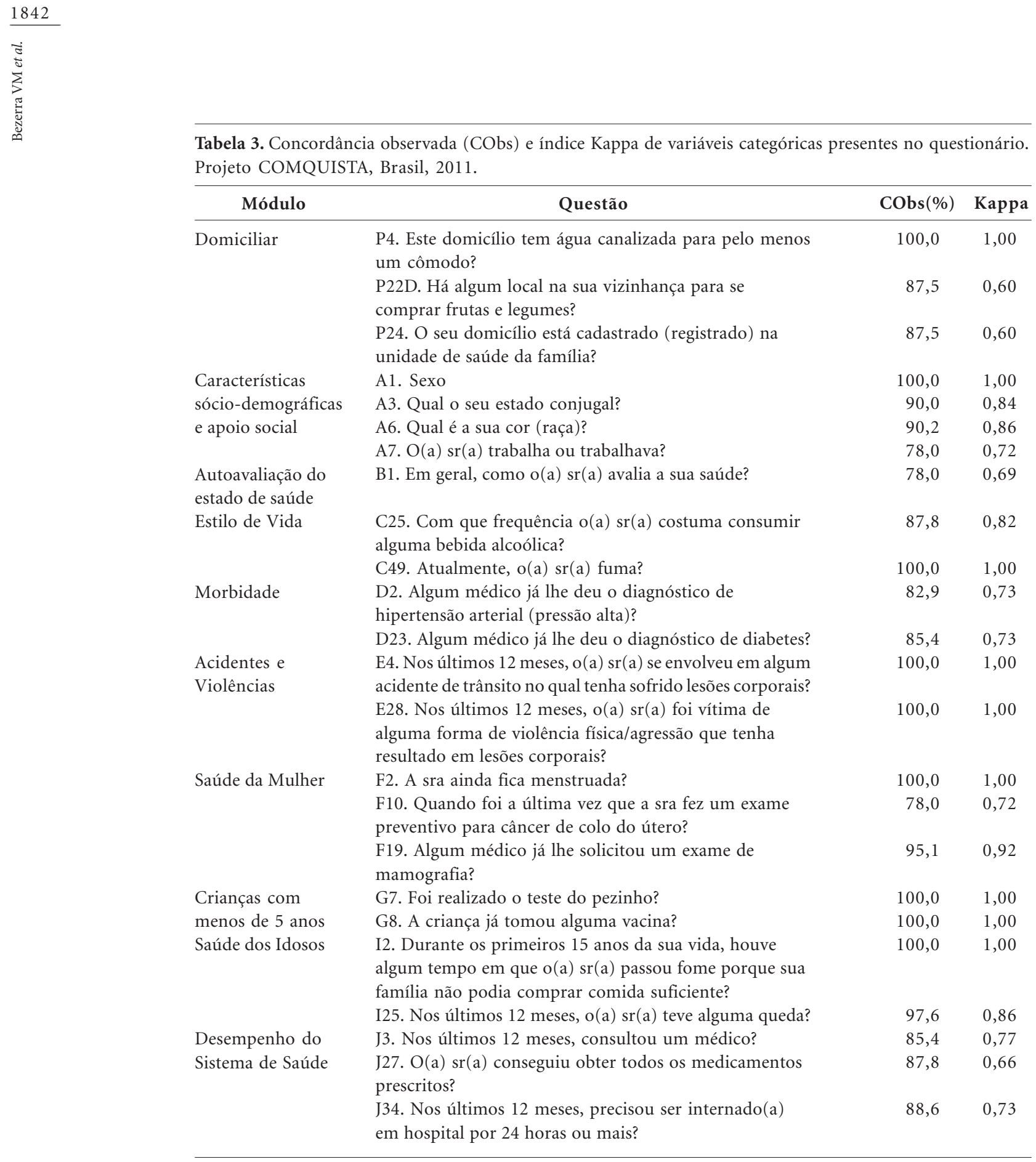

renda familiar per capita, mais da metade recebia até $1 / 4$ do salário-mínimo (Tabela 4).

Entre os adultos entrevistados, 54,3\% eram mulheres e mais da metade não trabalhava no momento da realização da pesquisa (Tabela 4). Observou-se que $41,5 \%$ apresentavam idade de 35 a 59 anos, a maioria era casada, com baixa escolaridade $(72,3 \%$ tinham até quatro anos completos de estudo) e referiu ter a cor parda $(44,7 \%)$ ou preta $(39,1 \%)$. Em relação às crianças, 50,8\% eram meninas e $38,0 \%$ tinham até dois anos de idade.

\section{Discussão}

Inquéritos populacionais são importantes para a construção de indicadores de saúde, assim como para uma melhor compreensão sobre os fatores de risco e os determinantes sociais do processo 
saúde-doença ${ }^{30}$. No entanto, uma das limitações geralmente observadas nas pesquisas nacionais é que o tamanho amostral não permite desagregações para níveis geográficos menores, tornando difícil a avaliação de grupos populacionais específicos, como as comunidades quilombolas.

O Projeto COMQUISTA foi direcionado aos quilombolas residentes em Vitória da Conquista/BA, reunindo dados que possibilitam uma maior compreensão dos determinantes de saúde dessa população. Um aspecto fundamental da pesquisa foi a utilização do questionário da PNS, o que permitirá futuras comparações dos resultados com características e indicadores nacionais.

A realização do estudo piloto foi essencial para verificar a adequação da dinâmica de recrutamento, testar os instrumentos de coleta de dados e confirmar a viabilidade da investigação. Além disso, orientou o ajuste do cálculo amostral e sinalizou a necessidade do desenvolvimento de estratégias de sensibilização e abordagem dos domicílios para reduzir as perdas. A análise da confiabilidade no piloto também possibilitou a adoção de medidas durante o treinamento dos entrevistadores que levaram ao aumento da qualidade dos dados obtidos no estudo principal.

$\mathrm{O}$ mapeamento permitiu a visualização espacial das comunidades e teve um papel fundamental na pesquisa de campo, já que a distribuição dos domicílios na zona rural não obedece à mesma lógica da zona urbana. Os mapas direcionaram o trabalho dos entrevistadores e facilitaram o planejamento da ação em cada comunidade. Destaca-se, ainda, a possibilidade do desenvolvimento de estudos geoespaciais a partir dos dados coletados.

No estudo principal a perda obtida foi aceitável e inferior à anteriormente sinalizada pelo piloto, o que demonstra a adequação das estratégias adotadas, entre as quais podemos destacar o agendamento de entrevistas para os finais de semana e o apoio dos ACS como agentes de divulgação do estudo. O resultado dessa ação foi percebido pelos entrevistadores durante a coleta de dados, já que na abordagem inicial dos domicílios a maioria dos moradores relatou ter obtido informações sobre a pesquisa.

O principal motivo das perdas foi não encontrar os indivíduos no domicílio após o procedimento padrão de três visitas, o que pode ser explicado pelo fato de se tratar de uma população residente em área rural e que costuma se deslocar para outras localidades a trabalho. Além disso, a perda foi diferencial para homens e indivíduos mais jovens, o que pode comprometer
Tabela 4. Características da população estudada. Projeto COMQUISTA, Brasil, 2011.

\begin{tabular}{cccc}
\hline \multicolumn{1}{c}{ Variáveis } & $\mathbf{N}$ & $\%$ & \\
\hline $\begin{array}{c}\text { Características dos domicílios } \\
\text { Abastecimento de água } \\
\text { Rede geral de abastecimento }\end{array}$ & & & \\
\hline & 9 & 2,3 &
\end{tabular}

Poço ou nascente

Cisterna $107 \quad 27,2$

Tanque/Açude/Aguada $\quad 156 \quad 39,7$

$\begin{array}{lll}\text { Outra } & 28 & 7,1\end{array}$

Esgotamento sanitário

Rede geral de esgoto ou fossa séptica $38 \quad \begin{array}{lll}38 & 9,7\end{array}$

Fossa rudimentar $\quad 161 \quad 41,1$

Outras formas $\quad 193 \quad 49,2$

Destino do lixo

Queimado ou enterrado

36793,4

Jogado no rio, terreno ou outro $26 \quad 6,6$

Forma de iluminação

Elétrica

Outras

Frequência de visitas do ACS

Mensal

Bimestral

1 a 4 vezes por ano

Nunca

$26 \quad 6,6$

37294,6

215,3

$196 \quad 50,5$

$44 \quad 11,3$

$48 \quad 12,4$

$100 \quad 25,8$

Renda familiar per capita

Até $1 / 4$ do salário mínimo $\quad 209 \quad 58,4$

$>$ 1/4 salário mínimo $\quad 149 \quad 41,6$

Características dos Adultos

Sexo

Masculino

$364 \quad 45,7$

Feminino

43354,3

Idade

18 a 34 anos

28936,3

35 a 59 anos

60 anos ou mais

$177 \quad 22,2$

Estado Conjugal

Casado ou vive com companheira $\quad 489 \quad 61,4$

$\begin{array}{lll}\text { Nunca casou/ Solteiro } & 187 & 23,5\end{array}$

Viúvo $\quad 64 \quad 8,0$

Separado/ Divorciado $\quad 57 \quad 7,2$

Escolaridade (anos completos de estudo)

Nunca estudou

$\begin{array}{lll}1 \text { a } 4 & 299 & 37,8\end{array}$

$274 \quad 34,6$

5 ou mais $219 \quad 27,7$

Cor autorreferida

Parda

$352 \quad 44,7$

$\begin{array}{lll}\text { Preta } & 308 & 39,1\end{array}$

$\begin{array}{lll}\text { Branca } & 100 & 12,7\end{array}$

Amarela $\quad 6 \quad 0,8$

$\begin{array}{lll}\text { Indígena } & 21 & 2,7\end{array}$

Situação de trabalho

Não trabalha atualmente $\quad 408 \quad 51,2$

$\begin{array}{lll}\text { Trabalha atualmente } & 389 & 48,8\end{array}$

ACS: Agente Comunitário de Saúde. ${ }^{* *}$ Salário mínimo vigente $=$ $\mathrm{R} \$ 545,00$. 
algumas estimativas. Perdas diferenciais em inquéritos geralmente são observadas nos estratos de renda mais elevada, devido à recusa, e nas áreas mais pobres dos grandes centros, onde a violência urbana é o principal motivo ${ }^{1}$.

A utilização de pockets foi considerada essencial, uma vez que possibilitou maior agilidade na aplicação das entrevistas e otimizou o trabalho de campo, eliminando a etapa de digitação e possíveis erros decorrentes da mesma. De modo geral, as estratégias utilizadas para garantia e controle de qualidade neste estudo foram capazes de gerar respostas confiáveis, podendo ser exemplificado através da análise de confiabilidade da variável "frequência de consumo de bebida alcoólica", que passou da classificação "razoável" no piloto para "quase perfeita" no estudo principal. A comparação entre a análise de confiabilidade do piloto e do estudo principal evidenciou a melhoria dos índices Kappa para a maioria das variáveis selecionadas.

Não obstante, o baixo coeficiente de correlação de Pearson apresentado pela variável "número de dias sem trabalhar por motivo de saúde" pode ser explicado pela influência temporal, pois o período ao qual a questão se referiu foi de 15 dias antecedentes à aplicação do questionário e as reentrevistas aconteciam até sete dias após a primeira entrevista. Assim, um valor baixo não significa menor confiabilidade da questão, mas que a situação pode ter mudado para os indivíduos no intervalo entre as entrevistas.

Quanto às características dos domicílios e aos serviços prestados, os quilombolas apresentaram maior cobertura de energia elétrica do que a população da zona rural do Brasil $(89,7 \%)$ e do Nordeste $(90,8 \%)$, porém menor do que a da população de Vitória da Conquista $(99,2 \%)$. O abastecimento de água por rede geral nessas comunidades foi muito inferior quando comparado ao da população rural brasileira $(27,8 \%)$, Nordeste $(34,9 \%)$ e de Vitória da Conquista $(87,3 \%)^{31,32}$. As principais formas de obtenção de água pelos quilombolas se davam por meio de tanques, açudes, poços ou cisternas, muitas vezes impróprias para uso. Já em relação ao esgotamento sanitário, o percentual de utilização de fossas rudimentares e de rede geral de esgoto ou fossa séptica pela população quilombola deste estudo foi semelhante ao da população rural do Brasil, do Nordeste e total de Vitória da Conquista $^{31,32}$.

Enquanto $89,1 \%$ da população de Vitória da Conquista, $26,9 \%$ da população rural do Brasil e $18,7 \%$ do Nordeste relataram a coleta de lixo re- gular como a principal forma de destino do $\operatorname{lixo}^{31,32}$, a maioria dos quilombolas queimava ou enterrava o lixo. Falta de infraestrutura de água, saneamento e gestão de resíduos sólidos adequados foi observada em outras pesquisas realizadas em comunidades quilombolas ${ }^{18-22}$ e populações indígenas no Brasil ${ }^{11}$, destacando fortemente a vulnerabilidade social que grupos étnico-raciais têm vivenciado no país.

Ao avaliar a frequência de visitas domiciliares pelos ACS, apenas 50,5\% das famílias quilombolas de Vitória da Conquista receberam uma visita ao mês, conforme orientação da portaria $\mathrm{n}^{\circ} 2.488^{33}$, o que demonstra que o cumprimento da recomendação ainda está distante de ser alcançado nesta população.

O perfil demográfico encontrado foi semelhante ao da população brasileira e de Vitória da Conquista quanto ao predomínio de mulheres, indivíduos com idade entre 35 a 59 anos e que tinham companheiros (as). Contudo, o percentual de quilombolas que se autodeclarou pretos $(39,1 \%)$ foi maior do que observado para o Brasil (6,9\%), Estado da Bahia (16,8\%), Região Metropolitana de Salvador $(29,4 \%)$ e mais especificamente, do que o município de Vitória da Conquista $(10,1 \%)^{31,32}$

A taxa de escolaridade dos quilombolas foi inferior à da população total de Vitória da Conquista $(78,8 \%)^{32}$, do Nordeste (59\%) e da zona rural do Brasil $(73,3 \%)^{31}$, sendo que a prevalência de indivíduos que nunca estudaram entre quilombolas foi maior do que a observada em populações indígenas $(15,2 \%)^{11}$. Quanto à renda familiar per capita, o percentual da população quilombola classificado na categoria de rendimentos de até $1 / 4$ do salário mínimo (valor vigente na época do estudo igual a $\mathrm{R} \$ 545,00)$ foi expressivamente maior que da população brasileira $(8,8 \%)$, do Nordeste $(19,8 \%)$ e de Vitória da Conquista $(5 \%)^{31,32}$. Destacando que as desigualdades vivenciadas pelos quilombolas podem ser observadas em nível regional.

O baixo nível socioeconômico associado ao isolamento geográfico das comunidades quilombolas tem sido apontado pelos estudos como alguns dos empecilhos para a melhoria das condições de vida e assistência à saúde aos indivíduos dessa populaçãa ${ }^{17,19-21}$.

\section{Considerações finais}

Os resultados deste inquérito demonstram inequívoca necessidade de avaliar as condições e os 
determinantes de saúde de populações vulneráveis no Brasil. A metodologia empregada foi considerada adequada para o alcance dos objetivos propostos, salientamos a bem sucedida adaptação e aplicação do questionário da PNS, o que deverá permitir comparações com conhecido grau de representatividade. O planejamento adequado, baseado na experiência do piloto, a coleta de dados por meio de pockets, a sensibilização prévia com ampla colaboração das lideranças locais, bem como o mapeamento, que possibilitou a localização espacial dos domicílios nas comunidades, são aspectos a serem ressaltados como positivos. Igualmente, a análise de confiabilidade de- monstrou a adequação das estratégias adotadas para garantia e controle de qualidade no estudo.

Os resultados preliminares apresentados sugerem a necessidade da implantação de ações para melhorar a qualidade de vida e reduzir o grau de vulnerabilidade da população quilombola de Vitória da Conquista. A exploração dos dados em análises específicas, incluindo uso e acesso aos serviços de saúde, morbidades, vulnerabilidade socioeconômica, dentre outros, devem gerar um amplo diagnóstico de saúde dos quilombolas, o que representa uma contribuição acadêmica de grande impacto e importância para esta população.

\section{Colaboradores}

VM Bezerra participou da elaboração do projeto, planejamento e coordenação da coleta dos dados, análises e redação do artigo. DS Medeiros e KO Gomes participaram da elaboração do projeto, planejamento e supervisão de campo, análises e redação do artigo. R Souzas, L Giatti e MDC Guimarães participaram da elaboração e orientação do projeto, planejamento da coleta dos dados e revisão final do artigo. AP Steffens, CN Kochergin, CL Souza, CS Moura, DA Soares, LRCS Santos, LGV Cardoso, MV Oliveira, PC Martins, OSC Neves participaram da elaboração do projeto, planejamento e supervisão da coleta de dados. 


\section{Agradecimentos}

Aos pesquisadores do Projeto COMQUISTA pela participação no planejamento e execução da pesquisa. À Secretaria Municipal de Saúde de Vitória da Conquista, especialmente aos profissionais de saúde responsáveis pelas comunidades quilombolas pesquisadas, pelo apoio oferecido para a realização do trabalho, e aos quilombolas entrevistados, pela receptividade.

Trabalho financiado pela Coordenação de Aperfeiçoamento de Pessoal de Nível Superior (CAPES), Doutorado Interinstitucional (DINTER), Ação novas fronteiras e pelo Ministério da Saúde/Conselho Nacional de Desenvolvimento Científico e Tecnológico/Fundação de Amparo à Pesquisa do Estado da Bahia/Secretaria de Saúde do Estado da Bahia (MS/CNPq/FAPESB/SESAB), Pesquisa Para o SUS: Gestão Compartilhada em Saúde (PPSUS-BA).

\section{Referências}

1. Waldman EA, Novaes HMD, Albuquerque MFM, Latorre MRDO, Ribeiro MCSA, Vasconcellos M, Ximenes RAA, Barata RB, Lago TG, Silva ZP. Inquéritos populacionais: aspectos metodológicos, operacionais e éticos. Rev Bras Epidemio 2008; 11(Supl. 1):168-179.

2. Laguardia J. O Uso da Variável "Raça" na Pesquisa em Saúde. Physis 2004; 14(2):197-234.

3. Bastos JL, Celeste RK, Faerstein E, Barros AJD. Racial discrimination and health: A systematic review of scales with a focus on their psychometric properties. Social Science \& Medicine 2010; 70(7):1091-1099.

4. Souzas R, Marinho OFP, Melo KLO. Acesso à saúde, promoção e prevenção ao HIV/AIDS e o recorte racial/étnico: revisão bibliográfica (1995 - 2009). In: Werneck J, Batista LE, Lopes F, organizadores. Saúde da população negra. Petrópolis, Brasília: ABPN; 2012. p. 266-286. Parte II.

5. Kabad JF, Bastos JL, Santos RV. Raça, cor e etnia em estudos epidemiológicos sobre populações brasileiras: revisão sistemática na base PubMed. Physis Rev Saúde Coletiva 2012; 22(3):895-918.

6. Coimbra Júnior CEA, Santos RV. Saúde, minorias e desigualdade: algumas teias de inter-relações, com ênfase nos povos indígenas no Brasil. Cien Saude Colet 2000; 5(1):125-132

7. Batista LE, Escuder MML, Pereira JCR. A cor da morte: causas de óbito segundo características de raça no Estado de São Paulo, 1999 a 2001. Rev Saude Publica 2004; 38(5):630-636.

8. Lopes F. Para além da barreira dos números: desigualdades raciais e saúde. Cad Saude Publica 2005; 21(5):1595-1601.

9. Cardoso AM, Santos RV, Coimbra Jr. CEA. Mortalidade infantil segundo raça/cor no Brasil: o que dizem os sistemas nacionais de informação? Cad Saude Publica 2005; 21(5):1602-1608.

10. Volochko A, Vidal NP. Desigualdades raciais na saúde: mortalidade nas regiões de saúde paulistas, 2005. Boletim do Instituto de Saúde 2010; 12(2):143-153.

11. Coimbra Júnior CE, Santos RV, Welch JR, Cardoso AM, Souza MC, Garnelo L, Rassi E, Follér ML, Horta BL. The First National Survey of Indigenous People's Health and Nutrition in Brazil: rationale, methodology, and overview of results. BMC Public Health 2013; 13(1):52.

12. Barata RB, Almeida MF, Montero CV, Silva ZP. Health inequalities based on ethnicity in individuals aged 15 to 64, Brazil, 1998. Cad Saude Publica 2007; 23(2):305-313.

13. Barata RB. Como e por que as desigualdades sociais fazem mal à saúde. Rio de Janeiro: Editora Fiocruz; 2009. 
14. Soares Filho MA. Vitimização por homicídios segundo características de raça no Brasil. Rev Saude Publica 2011; 45(4):745-755.

15. Guiotoku SK, Moysés ST, Moysés SJ, França BHS, Bisinelli JC. Iniquidades raciais em saúde bucal no Brasil. Rev Panam Salud Publica 2012; 31(2):135-141.

16. Brasil. Presidência da República. Casa Civil. Decreto no. 4887, de 20 de novembro de 2003. Regulamenta o procedimento para identificação, reconhecimento, delimitação, demarcação e titulação das terras ocupadas por remanescentes das comunidades dos quilombos de que trata o art. 68 do Ato das Disposições Constitucionais Transitórias. Diário Oficial da União 2003; 21 nov.

17. Silva JAN. Condições sanitárias e de saúde em Caiana dos Crioulos, uma comunidade Quilombola do Estado da Paraíba. Saúde e Soc 2007; 16(2):111-124.

18. Silva OS. Quilombos do Sul do Brasil: movimento social emergente na sociedade contemporânea. Rev Identidade 2010; 15(1):51-64.

19. Brasil. Ministério do Desenvolvimento Social e Combate à Fome. Chamada Nutricional Quilombola 2006. Brasília: Ministério do Desenvolvimento Social e Combate à Fome; 2006.

20. Volochko A, Batista LE. Saúde nos Quilombos. Temas em Saúde Coletiva 9. São Paulo: Instituto de Saúde-SESSP; 2009.

21. Guerrero AFH, Silva DO, Toledo LM, Guerrero JCH, Teixeira P. Mortalidade Infantil em Remanescentes de Quilombos do Município de Santarém Pará, Brasil. Saúde e Soc 2007; 16(2):103-110.

22. Pesquisa Nacional de Saúde PNS. Delineamento da PNS. [site da Internet] 2012. [acessado 2012 set 19]; [cerca de 1 p.]. Disponível em: http://www.pns. icict.fiocruz.br/index.php?pag= delineamento

23. Universidade Federal da Bahia (UFBA). Relatório técnico do Projeto COMQUISTA - Comunidades Quilombolas de Vitória da Conquista: Avaliação de Saúde e seus Condicionantes. Vitória da Conquista: UFBA, 2011. [site da Internet] 2012. [acessado $2012 \mathrm{fev} 22$ ]; [cerca de 41 p.]. Disponível em: http://projeto comquista.wordpress.com.

24. Fundação Palmares. Comunidades Quilombolas de Vitória da Conquista, Bahia. [site da Internet] 2010. [acessado 2010 ago 23]; [cerca de 4 p.]. Disponível em: http://www.palmares.gov.br/quilombola/?estado $=$ BA.

25. Vitória da Conquista. Secretaria Municipal de Saúde (SMS). Informações sobre a Gestão do Sistema de Atenção Básica e composição das Equipes de Saúde da Família 2010. Vitória da Conquista: SMS; 2010. [dados não publicados].
26. Sherman GE, Sutton T, Blazek R, Holl S, Dassau O, Morely B, Mitchell T, Luthman L. Quantum GIS User Guide - Version 1.7 "Wroclaw". [site da Internet] 2011. [acessado 2011 jun 12]; [cerca de 207 p.]. Disponível em: http://download.osgeo.org/qgis/ doc/manual/qgis-1.7.0_user_guide_en.pdf.

27. Jelliffe DB. The assessment of nutritional status of the community. Geneva: WHO; 1966.

28. Sociedade Brasileira de Cardiologia (SBC). VI Diretrizes de Hipertensão Arterial da Sociedade Brasileira de Cardiologia (2010). Arq Bras de Card 2010; 17(1 Supl. 1):1-69.

29. Landis JR, Koch GG. The measurement of observer agreement for categorical data. Biometrics 1977; 33(1):159-174.

30. Viacava F. Informações em saúde: a importância dos inquéritos populacionais. Cien Saude Colet 2002; 7(4):607-621.

31. Instituto Brasileiro de Geografia e Estatística (IBGE). Censo Demográfico 2010. Características da população e dos domicílios. Resultados do universo. Rio de Janeiro: IBGE; 2011.

32. Instituto Brasileiro de Geografia e Estatística (IBGE) Censo Demográfico 2010. Características da populacão e dos domicílios: Resultados do Universo. [site da Internet] 2013 [acessado $2013 \mathrm{fev} 21$ ]; [cerca de 8 p.]. Disponível em: http://www.ibge.gov.br/cidadesat/topwindow.htm? 1

33. Brasil. Ministério da Saúde (MS). Portaria GM/MS $n^{\circ} 2.488$, de 21 de outubro de 2011. Aprova a Política Nacional de Atenção Básica, estabelecendo a revisão de diretrizes e normas para a organização da Atenção Básica, para a Estratégia Saúde da Família (ESF) e o Programa de Agentes Comunitários de Saúde (PACS). Diário Oficial da União 2011; 22 out.

Artigo apresentado em 15/03/2013

Aprovado em 23/03/2013

Versão final apresentada em 24/03/2013 
